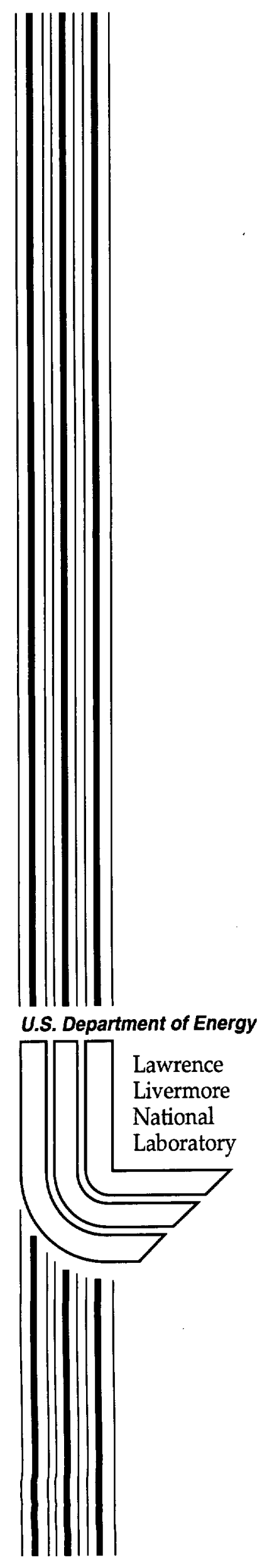

UCRL-ID-145502

\title{
Hydrodynamic Theory of Atomic Mixing in Multicomponent Gases and Plasmas
}

\author{
J.D. Ramshaw
}

August 22, 2001 


\section{DISCLAIMER}

This document was prepared as an account of work sponsored by an agency of the United States Government. Neither the United States Government nor the University of California nor any of their employees, makes any warranty, express or implied, or assumes any legal liability or responsibility for the accuracy, completeness, or usefulness of any information, apparatus, product, or process disclosed, or represents that its use would not infringe privately owned rights. Reference herein to any specific commercial product, process, or service by trade name, trademark, manufacturer, or otherwise, does not necessarily constitute or imply its endorsement, recommendation, or favoring by the United States Government or the University of California. The views and opinions of authors expressed herein do not necessarily state or reflect those of the United States Government or the University of California, and shall not be used for advertising or product endorsement purposes.

This is a preprint of a paper intended for publication in a journal or proceedings. Since changes may be made before publication, this preprint is made available with the understanding that it will not be cited or reproduced without the permission of the author.

This report has been reproduced directly from the best available copy.

Available electronically at http://www.doe.gov/bridge

Available for a processing fee to U.S. Department of Energy

and its contractors in paper from

U.S. Department of Energy

Office of Scientific and Technical Information

P.O. Box 62

Oak Ridge, TN 37831-0062

Telephone: (865) 576-8401

Facsimile: (865) 576-5728

E-mail: reports@adonis.osti.gov

Available for the sale to the public from

U.S. Department of Commerce

National Technical Information Service

5285 Port Royal Road

Springfield, VA 22161

Telephone: (800) 553-6847

Facsimile: (703) 605-6900

E-mail: orders@ntis.fedworld.gov

Online ordering: http://www.ntis.gov/ordering.htm

OR

Lawrence Livermore National Laboratory

Technical Information Department's Digital Library

http://www.llnl.gov/tid/Library.html 


\title{
HYDRODYNAMIC THEORY OF ATOMIC MIXING \\ IN MULTICOMPONENT GASES AND PLASMAS *
}

J. D. Ramshaw

\begin{abstract}
Atomic mixing in multicomponent gases and plasmas is usually described as a diffusional process. The diffusional description is an approximation to a more general dynamical description in which the motion of each individual species or material is governed by its own momentum equation, with appropriate coupling terms to represent the exchange of momentum between different species. These equations are not new, but they are scattered in the literature. Here we summarize the form of these species momentum equations, and the coupling coefficients therein, in sufficient detail to facilitate their inclusion and use to simulate atomic mixing in hydrodynamics codes.
\end{abstract}

*This work was performed under the auspices of the U.S. Department of Energy by the University of California Lawrence Livermore National Laboratory under contract No. W-7405-Eng-48. 


\section{INTRODUCTION}

The dynamics of multicomponent gases and plasmas is usually described in terms of momentum and energy transport equations for the fluid mixture as a whole, continuity equations for each species or component in the mixture, and constitutive relations for the fluxes of momentum, energy, and species masses due to molecular collisions. These constitutive relations are ordinarily diffusional in character. For such a description to be valid, the collisional transfer of momentum and/or energy between the different species must be fast relative to time scales of interest. When this condition is not satisfied, it becomes necessary to use a more general description in which each species has its own momentum and/or energy transport equation. The conventional diffusional description of species transport is simply an approximation to these individual species momentum equations $[1,2]$.

In order to simulate the atomic mixing of materials in fast processes where the diffusion approximation is not valid, it is necessary to solve the individual species momentum equations. This in turn requires the evaluation of the various coupling terms and coefficients therein. These equations are not new, but they are scattered in the literature. Our purpose here is to facilitate their future use and application by gathering them together and sum.marizing them in sufficient detail and completeness to serve as a guide or blueprint for their incorporation into hydrodynamics codes. All equations are written in cgs units.

In their most general form, the species momentum equations allow each species to have its own temperature [1]. However, this degree of generality is rarely required, and the present discussion will be restricted to partially ionized multicomponent plasmas containing neutral atoms (or molecules), ions, and free electrons, in which all the heavy-particle species 
have the same common temperature $T$, while the electrons may have a different temperature $T_{e}$. Neutral gases containing no free electrons or ions are simply a special case. The plasma is assumed to be unmagnetized and electrically neutral, so magnetic field effects [2] will be neglected. However, a spontaneous electric field $\mathbf{E}$ will nevertheless arise to preserve local charge neutrality, and it is essential to include the resulting electrical forces in the momentum equations for the charged species.

\section{SPECIES CONTINUITY EQUATIONS AND MASS FLUXES}

In the absence of chemical reactions or other mass exchange between species, the continuity or mass conservation equation for species $i$ is simply

$$
\frac{\partial \rho_{i}}{\partial t}+\nabla \cdot\left(\rho_{i} \mathbf{u}_{i}\right)=0
$$

where $\rho_{i}$ and and $\mathbf{u}_{i}$ are respectively the partial mass density and mean velocity of species $i$. Summing over $i$ gives the total continuity equation for the mixture:

$$
\frac{\partial \rho}{\partial t}+\nabla \cdot(\rho \mathbf{u})=0
$$

where $\rho=\sum_{i} \rho_{i}$ is the total mass density of the mixture, $\mathbf{u}=\sum_{i} y_{i} \mathbf{u}_{i}$ is the mass-weighted mean fluid velocity, and $y_{i}=\rho_{i} / \rho$ is the mass fraction of species $i$. Equation (1) is usually written in the form

$$
\frac{\partial \rho_{i}}{\partial t}+\nabla \cdot\left(\rho_{i} \mathbf{u}\right)=-\nabla \cdot \mathbf{J}_{i}
$$


where $\mathbf{J}_{i}=\rho_{i} \mathbf{w}_{i}$ and $\mathbf{w}_{i}=\mathbf{u}_{i}-\mathbf{u}$. The number density of species $i$ is denoted by $n_{i}=\rho_{i} / m_{i}$, where $m_{i}$ is the mass of a single particle of species $i$.

The total charge density in the plasma is $\rho_{q}=\sum_{i} \rho_{i} q_{i}$, where $q_{i}$ is the charge per unit mass of species $i$. An evolution equation for $\rho_{q}$ may be derived by multiplying Eq. (1) by $q_{i}$ and summing over $i$ to obtain

$$
\frac{\partial \rho_{q}}{\partial t}+\nabla \cdot \mathbf{J}_{q}=0
$$

where $\mathrm{J}_{q}=\sum_{i} \rho_{i} q_{i} \mathbf{u}_{i}$ is the electrical current density. This is simply the usual charge conservation equation, which is also implied by Maxwell's equations. Since it is a linear combination of the continuity equations for the charged species, it can be used to replace any one of those equations, in particular that for the free electrons. In the present context, however, it is unnecessary to actually solve this equation, because our restriction to electrically neutral plasmas implies that $\rho_{q}=0$, so that

$$
\rho_{e}=-\frac{1}{q_{e}} \sum_{j \neq e} \rho_{j} q_{j}
$$

where the species index $i=e$ refers to the free electrons. This equation determines the electron mass density $\rho_{e}$ and number density $n_{e}=\rho_{e} / m_{e}$ in terms of the heavy-particle species densities. It is therefore unnecessary to explicitly solve Eq. (1) for $i=e$, so this equation is not needed and can be ignored. We therefore retain Eq. (1) only for $i \neq e$. 


\section{SPECIES MOMENTUM EQUATIONS}

Neglecting viscous stresses, the momentum equation for species $i$ takes the form [1]

$$
\frac{\partial\left(\rho_{i} \mathbf{u}_{i}\right)}{\partial t}+\nabla \cdot\left(\rho_{i} \mathbf{u}_{i} \mathbf{u}_{i}\right)=-\nabla p_{i}+\rho_{i} \mathbf{F}_{i}+\sum_{j} \mathbf{F}_{i j}
$$

where $p_{i}$ is the partial pressure of species $i, \mathbf{F}_{i}$ is the external body force per unit mass acting on species $i$, and $\mathbf{F}_{i j}=-\mathbf{F}_{j i}$ is the mean force per unit volume of species $j$ on species $i$. In the present context the only body forces are gravity and the electric forces on the charged species, so that $[2,3]$

$$
\mathbf{F}_{i}=\mathbf{g}+q_{i} \mathbf{E}
$$

where $\mathbf{g}$ is the acceleration of gravity. The species interaction forces are of the form [1]

$$
\mathbf{F}_{i j}=\alpha_{i j}\left(\mathbf{u}_{j}-\mathbf{u}_{i}\right)+\beta_{i j} \nabla \ln T_{j}-\beta_{j i} \nabla \ln T_{i}
$$

where $\alpha_{i j}=\alpha_{j i}$, and $T_{i}=T$ if $i \neq e$. The form of $\alpha_{i j}$ and $\beta_{i j}$ will be given in the next section. Although we will not make use of the diffusion approximation here, we note in passing that the binary diffusivities for species pairs are simply related to the friction coefficients by $D_{i j}=p_{i} p_{j} /\left(p \alpha_{i j}\right)$, where $p=\sum_{i} p_{i}$ is the total pressure [1].

The free electrons are much lighter than the other species, and they consequently respond to and equilibrate with the various forces much more quickly than the heavy particles do. This occurs on very short time scales associated with the small value of $m_{e}$. If the equations were solved using an explicit numerical scheme, these short electron time scales would present unacceptable stability and/or accuracy restrictions on the time step. These 
restrictions can be analytically circumvented by systematically neglecting terms of order $\sqrt{m_{e}}$ and $m_{e}$ in Eq. (6), which also simplifies the equations. For this purpose we need to know the orders of magnitude of various quantities in terms of $m_{e}$, in particular: $\rho_{e}=m_{e} n_{e} \sim m_{e}$, $q_{e} \sim m_{e}^{-1}, \alpha_{j e} \sim \sqrt{m_{e}}[2]$, and $\beta_{e j} \ll \beta_{j e}$ [4]. Neglecting terms of order $\sqrt{m_{e}}$ and higher in the electron momentum equation, we then obtain

$$
\mathbf{E}=\frac{1}{\rho_{e} q_{e}}\left[\nabla p_{e}+\left(\sum_{j \neq e} \beta_{j e}\right) \nabla \ln T_{e}\right]
$$

which now replaces Eq. (6) for $i=e$. This equation explicitly determines $\mathbf{E}$, but it does not determine the electron velocity $\mathbf{u}_{e}$. However, the neutrality condition $\rho_{q}=0$ implies that $\nabla \cdot \mathbf{J}_{q}=0$, and we shall in fact assume the stronger "ambipolar" condition $\mathbf{J}_{q}=0$, which also follows from Ampere's law in the MHD approximation $[2,3]$. This then implies that

$$
\rho_{e} q_{e} \mathbf{u}_{e}=-\sum_{j \neq e} \rho_{j} q_{j} \mathbf{u}_{j}
$$

which determines $\mathbf{u}_{e}$ in terms of the velocities of the heavy species.

For consistency we also neglect terms of order $\sqrt{m_{e}}$ and higher in the heavy-particle momentum equations, which then become

$$
\begin{aligned}
\frac{\partial\left(\rho_{i} \mathbf{u}_{i}\right)}{\partial t}+\nabla \cdot\left(\rho_{i} \mathbf{u}_{i} \mathbf{u}_{i}\right) & =-\nabla p_{i}+\rho_{i}\left(\mathbf{g}+q_{i} \mathbf{E}\right)+\sum_{j \neq i, e} \alpha_{i j}\left(\mathbf{u}_{j}-\mathbf{u}_{i}\right) \\
& +\left[\sum_{j \neq i, e}\left(\beta_{i j}-\beta_{j i}\right)\right] \nabla \ln T+\beta_{i e} \nabla \ln T_{e}
\end{aligned}
$$

where $i \neq e$. The net result of these simplifications is that the continuity and momentum 
Eqs. (1) and (11) need only be solved for $i \neq e$, with $\mathrm{E}$ given by Eq. (9), while $\rho_{e}$ and $\mathbf{u}_{e}$ are determined by Eqs. (5) and (10), respectively.

\section{THE COUPLING COEFFICIENTS}

We must now define the coefficients $\alpha_{i j}$ and $\beta_{i j}$, which is unfortunately a rather tedious proposition. Equation (11) shows that we need both these coefficients for all heavyspecies pairs, as well as the coefficients $\beta_{i e}$ for all heavy species $i$. In what follows it will be understood that the subscripts $i$ and $j$ both refer to heavy species. The central quantity required to compute both $\alpha_{i j}$ and $\beta_{i j}$ is a collision integral denoted by $\Omega_{i j}^{(1)}(1, T)[5,6]$, in terms of which $[1,4]$

$$
\begin{aligned}
& \alpha_{i j}=(16 / 3) n_{i} n_{j} \mu_{i j} \Omega_{i j}^{(1)}(1, T) \\
& \beta_{i j}=-(16 / 3)\left(\tau_{j} / m_{j}^{2}\right) \mu_{i j}^{2} n_{i} n_{j} k_{B} T^{2} \partial \Omega_{i j}^{(1)}(1, T) / \partial T
\end{aligned}
$$

where $k_{B}$ is Boltzmann's constant, $\mu_{i j}=m_{i} m_{j} /\left(m_{i}+m_{j}\right)$, and the collision time $\tau_{i}$ is given by $[4]$

$$
\tau_{i}=\sqrt{\frac{\pi}{8 k_{B} T}}\left(\sum_{j \neq e} \frac{n_{j} \sigma_{i j}}{\sqrt{\mu_{i j}}}\right)^{-1}
$$

where the cross-sections $\sigma_{i j}$ are given by

$$
\sigma_{i j}=\left(\frac{2 \pi \mu_{i j}}{k_{B} T}\right)^{1 / 2} \Omega_{i j}^{(1)}(1, T)
$$

These cross-sections differ by a factor of $3 / 4$ from the average momentum transfer cross- 
sections previously employed [4]. The definition of Eq. (15) now seems preferable, as it properly reduces to the correct geometrical cross-sections for hard spheres [1].

The collision integrals $\Omega_{i j}^{(1)}(1, T)$ for neutral pairs are obtained from a standard functional fit in terms of the Lennard-Jones parameters $\sigma_{i}$ and $\epsilon_{i}$ for species $i[7]$,

$$
\Omega_{i j}^{(1)}(1, T)=\sigma_{i j}\left(\frac{k_{B} T}{2 \pi \mu_{i j}}\right)^{1 / 2} \Omega^{*}\left(k_{B} T / \epsilon_{i j}\right)
$$

where $\sigma_{i j}=(\pi / 4)\left(\sigma_{i}+\sigma_{j}\right)^{2}, \epsilon_{i j}=\sqrt{\epsilon_{i} \epsilon_{j}}$, and $\Omega^{*}(x)=x^{-0.145}+4(1+2 x)^{-2}$. The collision integrals for charged species pairs are given by $[5,6,8-10]$

$$
\Omega_{i j}^{(1)}(1, T)=\left(\frac{\pi}{\mu_{i j}}\right)^{1 / 2} \frac{\left(Q_{i} Q_{j}\right)^{2}}{\left(2 k_{B} T\right)^{3 / 2}} \ln \left(1+\Lambda_{i j}^{2}\right)^{1 / 2}
$$

where $Q_{i}=m_{i} q_{i}$ is the charge on a single particle of species $i, \Lambda_{i j}=3 k_{B} T b_{i j}^{0} /\left|Q_{i} Q_{j}\right|$, and $b_{i j}^{0}$ is the maximum allowed impact parameter for Coulomb collisions of an $i j$ pair. The use of such a cutoff is an inherently approximate procedure, and there is some uncertainty about the appropriate choice of $b_{i j}^{0}$ [6]. Nowadays it is customary to identify $b_{i j}^{0}$ with the Debye length $\lambda_{D}$, but this is sensible only when the latter is much larger than the mean interparticle . spacing for species pair $i j$, which may be estimated as $\bar{r}_{i j}=\left[\max \left(n_{i}, n_{j}\right)\right]^{-1 / 3}$. We therefore let $b_{i j}^{0}=\max \left(\lambda_{D}, \bar{r}_{i j}\right)$.

There is some further uncertainty as to the appropriate Debye length to use for this purpose. The obvious choice is the full multicomponent Debye length given by [8]

$$
\lambda_{D}=\frac{1}{2 \sqrt{\pi}}\left(\frac{n_{e} Q_{e}^{2}}{k_{B} T_{e}}+\sum_{i \neq e} \frac{n_{i} Q_{i}^{2}}{k_{B} T}\right)^{-1 / 2}
$$


However, the present equations are primarily intended for use in fast dynamical problems with short time scales, where one intuitively feels that Debye shielding will be mainly due to the electrons [11]. This suggests that the shielding due to ions should be neglected, which can be done by omitting the ion terms in Eq. (18) to obtain

$$
\lambda_{D}=\left(\frac{k_{B} T_{e}}{4 \pi n_{e} Q_{e}^{2}}\right)^{1 / 2}
$$

Moreover, the essential effect of electron shielding is that an ion of species $i$ viewed from a distance $r$ appears to have a reduced effective charge of $Q_{i} \exp \left(-r / \lambda_{D}\right)$. On this basis, one might further argue that the the effective interaction potential between two such ions is thereby reduced by a factor $\exp \left(-2 r / \lambda_{D}\right)$, so that a factor of $1 / 2$ should be inserted into the right member of Eq. (19) for present purposes. These ambiguities are troublesome and unsatisfactory, but fortunately they occur in the argument of a logarithm and consequently correspond to a relatively minor uncertainty in the value of $\Omega_{i j}^{(1)}(1, T)$.

The collision integrals for charged-neutral species pairs interacting via a pair potential $\phi_{i j}=-(1 / 2)\left(Q^{2} \alpha_{p}\right)_{i j} / r_{i j}^{4}$ are given by $[5,6]$

$$
\Omega_{i j}^{(1)}(1, T)=0.703\left[\frac{\left(Q^{2} \alpha_{p}\right)_{i j}}{\mu_{i j}}\right]^{1 / 2}
$$

where $\left(Q^{2} \alpha_{p}\right)_{i j}$ is the polarizability of the neutral species times the square of the charge on the charged species. However, use of Eq. (20) is tantamount to assuming that the collision integrals for charged-neutral pairs are dominated by the asymptotic long-range attractive part of the interparticle potential rather than the short-range repulsive part, and this seems 
questionable. It might therefore be preferable to simply approximate the collision integrals for such pairs by the hard-sphere formula $\Omega_{i j}^{(1)}(1, T)=R_{i j}^{2}\left(\pi k_{B} T / 2 \mu_{i j}\right)^{1 / 2}$ instead, where $R_{i j}$ is some reasonable estimate of the distance of closest approach of an $i j$ pair.

Finally, the thermal coupling coefficients $\beta_{i e}$ are given by [4]

$$
\beta_{i e}=\frac{3}{2} k_{B} T_{e}\left(\frac{n_{i} Z_{i}^{2}}{\bar{Z}+\sqrt{2}}\right)
$$

where $Z_{i}=Q_{i} / Q_{e}, n_{e} \bar{Z}=\sum_{j \neq e} n_{j} Z_{j}^{2}$, and we have neglected a summation over neutral species which is generally expected to be small [4]. Note that $\beta_{i e}$ vanishes for neutral molecules in this approximation [4].

\section{MODIFICATIONS TO THE HYDRODYNAMIC EQUATIONS}

The relative motion of the different species or materials in the mixture gives rise to certain additional terms in the momentum and energy transport equations for the mixture as a whole [12]. If it is desired to implement the present equations into existing hydrodynamics codes, these additional terms should be introduced into the existing momentum and energy . equations that such codes already contain.

The relative species motion gives rise to an additional stress tensor of the form [12]

$$
\mathrm{R}=-\sum_{i} \rho_{i} \mathbf{w}_{i} \mathbf{w}_{i}
$$

which must be introduced into both the momentum equation and the work terms in the energy equation. In the momentum equation, this additional stress is formally equivalent to 
an additional body force per unit volume of $\nabla \cdot R$.

The relative species motion also introduces additional heat fluxes into the energy equation, namely [12]

$$
\begin{aligned}
& \mathbf{J}_{q}=\sum_{i} \frac{1}{2}\left|\mathbf{w}_{i}\right|^{2} \mathbf{J}_{i} \\
& \mathbf{J}_{h}=\sum_{i} h_{i} \mathbf{J}_{i}
\end{aligned}
$$

where $h_{i}=e_{i}+p_{i} / \rho_{i}$ is the specific thermal enthalpy of species $i$. These heat fluxes are formally equivalent to an energy source per unit volume per unit time of $-\nabla \cdot\left(\mathbf{J}_{q}+\mathbf{J}_{h}\right)$.

The relative species motion further implies an additional kinetic energy per unit volume of $\rho q$, where [12]

$$
q=\sum_{i} \frac{1}{2} y_{i}\left|\mathbf{w}_{i}\right|^{2}
$$

However, it is essential to note that this energy is not contained in the mixture kinetic energy density $\frac{1}{2} \rho|\mathbf{u}|^{2}$, so it effectively constitutes a second non-thermal type of internal energy. The total energy per unit mass of the mixture (exclusive of gravitational or electromagnetic field energy) is consequently given by [12]

$$
E=\frac{1}{2}|\mathbf{u}|^{2}+q+e
$$

where $e$ is the specific thermal internal energy per unit mass, which of course is the energy that enters into thermodynamic state relations. It follows that in existing computer codes 
where the specific internal energy is defined in the usual way, namely

$$
\varepsilon \equiv E-\frac{1}{2}|\mathbf{u}|^{2}
$$

it now becomes necessary to reinterpret this energy as $e+q$, so that $q$ must be subtracted from $\varepsilon$ to obtain the purely thermal internal energy $e$ required for thermodynamic state equation purposes.

Most of the above modifications become unnecessary if it is assumed that the relative species velocities $\mathbf{w}_{i}$ are sufficiently small that terms of order $\left|\mathbf{w}_{i}\right|^{2}$ may be neglected. Indeed, the only surviving modification under that assumption is the additional heat flux $\mathbf{J}_{h}$ in the energy equation. Unfortunately, the validity of this approximation will rarely be obvious a priori, so it would be unwise to adopt it as a general procedure, except perhaps on a provisional basis pending later verification.

\section{NUMERICAL CONSIDERATIONS}

\subsection{Consistency with Total Momentum}

If it is desired to implement the present equations into existing hydrodynamics codes, we must consider how to deal with the existing momentum equations that such codes already contain. We can of course sum Eq. (6) over $i$ to obtain an evolution equation for the total momentum density $\rho \mathbf{u}=\sum_{i} \rho_{i} \mathbf{u}_{i}$, but the result may not be fully consistent with the preexisting momentum equation in the code for several reasons, including (a) our neglect of terms of order $\sqrt{m_{e}}$ and higher, (b) our neglect of viscous terms in the species momentum 
equations, and (c) any differences between the numerical scheme used to solve the species momentum equations and that used to solve the total momentum equation in the code. In principle it would be desirable to eliminate all such inconsistencies, but in practice this may not always be feasible. In such cases, the species velocities computed by Eqs. (10) and (11) should be suitably corrected to force them to be consistent with the mean fluid velocity $\mathbf{u}$ computed by the existing momentum equation already in the code. This can be done by means of a procedure originally developed to repair inconsistent.diffusion fluxes [13]. Let the uncorrected species velocities computed by Eqs. (10) and (11) be denoted by $\mathbf{u}_{i}^{0}$. These velocities are then immediately replaced, on every time step, by the corrected species velocities

$$
\mathbf{u}_{i}=\mathbf{u}+\mathbf{u}_{i}^{0}-\sum_{j} y_{j} \mathbf{u}_{j}^{0}
$$

These corrected velocities now obey the constraint $\sum_{i} \rho_{i} \mathbf{u}_{i}=\rho \mathbf{u}$ as they should, which in turn ensures that the total density $\rho=\sum_{i} \rho_{i}$ computed by Eqs. (1) and (5) will automatically be consistent (within roundoff error) with that produced by the existing continuity equation already in the code, provided of course that Eq. (1) is solved by the same temporal and spatial difference scheme. The correction procedure of Eq. (28) is equivalent to using Eqs. (10) and . (11) to determine the relative species velocities only, while $\mathbf{u}$ is obtained from the existing momentum equation already in the code [13]. It is easy to verify that the resulting corrected species velocities still satisfy Eq. (10).

An alternative way to ensure consistency between the species velocities $\mathbf{u}_{i}$ and the mass-weighted mean velocity $\mathbf{u}$ is to algebraically convert Eqs. (10) and (11) into evolution equations for $\mathbf{w}_{i}$ rather than $\mathbf{u}_{i}$. This has two advantages: (a) it directly computes the 
quantity which is most often needed, and (b) it avoids the loss of significant figures that would occur in computing small values of $\mathbf{w}_{i}$ by subtracting nearly equal values of $\mathbf{u}_{i}$ and $\mathbf{u}$. In places where $\mathbf{u}_{i}$ itself is required, it may simply be evaluated as $\mathbf{u}+\mathbf{w}_{i}$. The disadvantages are that (a) $\mathbf{w}_{i}$ is less closely related to a conserved quantity than $\mathbf{u}_{i}$, and (b) the evolution equation for $\mathbf{w}_{i}$ is slightly more complicated than that for $\mathbf{u}_{i}$.

The conversion of Eqs. (10) and (11) into evolution equations for $\mathbf{w}_{i}$ is straightforward but tedious, with the final results

$$
\begin{aligned}
\rho_{e} q_{e} \mathbf{w}_{e} & =-\sum_{j \neq e} \rho_{j} q_{j} \mathbf{w}_{j} \\
\rho_{i} \frac{D \mathbf{w}_{i}}{D t} & =-\nabla p_{i}+y_{i} \nabla p+\rho_{i} q_{i} \mathbf{E}-\rho_{i} \mathbf{w}_{i} \cdot \nabla\left(\mathbf{w}_{i}+\mathbf{u}\right)-y_{i} \nabla \cdot \mathrm{R} \\
& +\sum_{j \neq i, e} \alpha_{i j}\left(\mathbf{w}_{j}-\mathbf{w}_{i}\right)+\left[\sum_{j \neq i, e}\left(\beta_{i j}-\beta_{j i}\right)\right] \nabla \ln T+\beta_{i e} \nabla \ln T_{e} \quad(i \neq e)
\end{aligned}
$$

where $D / D t=\partial / \partial t+\mathbf{u} \cdot \nabla$. In principle these equations should preserve the identity $\sum_{j} \rho_{j} \mathbf{w}_{j}=0$ to within roundoff errors. However, it would be prudent to prevent any possible accumulation of such errors by enforcing that identity as a constraint by means of a corrective procedure analogous to Eq. (28), viz.

$$
\mathbf{w}_{i}=\mathbf{w}_{i}^{0}-\sum_{j} y_{j} \mathbf{w}_{j}^{0}
$$

\subsection{Time Differencing}

The simplest time differencing scheme that might be used is to simply (a) approximate all time derivatives $d Q / d t$ by $\left(Q^{n+1}-Q^{n}\right) / \Delta t$, where $Q^{n}$ is the difference approximation to $Q\left(t_{n}\right)$ and $\Delta t=t_{n+1}-t_{n}$ is the time step, and (b) evaluate the remaining quantities in the 
equations as $Q^{n}$, or preferably $Q^{n+1}$ if the latter has already been calculated. A natural solution sequence for the present equations would be the following:

- Solve Eqs. (29) and (30), or Eqs. (10) and (11), to obtain $\mathbf{w}_{i}^{n+1}$ or $\mathbf{u}_{i}^{n+1}$;

- Correct the resulting values of $\mathbf{w}_{i}^{n+1}$ or $\mathbf{u}_{i}^{n+1}$ by means of Eq. (31) or (28);

- Use the resulting new-time values of $\mathbf{w}_{i}^{n+1}$ or $\mathbf{u}_{i}^{n+1}$ in Eqs. (1) or (3) to solve these equations and Eq. (5) for $\rho_{i}^{n+1}$;

- Evaluate the additional terms discussed in Sect. 5 above and add their contributions into the momentum and energy equations for the mixture as a whole.

This scheme would of course be first-order accurate in time, but it would be straightforward to modify it into a two-step second-order predictor-corrector scheme if desired.

The explicit time differencing of the frictional coupling terms in Eq. (11) or (30) imposes new stability restrictions on the time step [14], and these restrictions must be incorporated into the time step control logic in the code. In some cases these new stability restrictions may require the use of unacceptably small time steps, but this can be ameliorated in the usual way by subcycling. Alternatively, these restrictions can be eliminated by using an implicit scheme instead [14], but this would require inversion of an $N \times N$ matrix on every zone of the computing mesh, where $N$ is the number of heavy species.

\subsection{Space Differencing}

A suitable stable upwinding scheme must of course be selected for the convection terms in Eqs. (1) or (3) and (11) or (30). In Eulerian codes it would be natural to use the 
same convective scheme used for the other equations in the code, whereas the choice of a convection scheme represents a new decision that must be made to implement the present equations in Lagrangian codes. The corresponding convective Courant stability restrictions on the time step must of course also be incorporated into the time step control logic.

\subsection{Trace Species}

All species are not always present at all points in the flow field, so it is necessary to allow for the fact that some of the species densities at any given point may be zero. In particular, logic must be included to prevent dividing by $\rho_{i}$ when it is small or zero. If Eq. (11) is solved in conservative form, it should yield $\rho_{i} \mathbf{u}_{i}=0$ in regions where species $i$ is absent, since such a species can have no momentum. The value of $\mathbf{u}_{i}$ is then indeterminate, but the choice of this indeterminate value should have no physical consequences, and care must be taken to ensure that this is the case.

\subsection{Composite Species}

In practical calculations, it will not always be feasible to consider every species present as a separate species with its own momentum equation. In such situations, it is necessary to combine or lump together several species into a single composite species. For example, one may wish to lump together some or all of the isotopes and/or ionization states of a particular element to obtain a single composite species representing that element. This discards information about the relative velocities of the species being lumped together, so these species must be regarded as all having the same velocity. It is then easy to verify that the resulting composite species obey continuity and momentum equations of the same 
form as those already given for individual species, provided that the coupling coefficients and charge per unit mass are evaluated as appropriate sums over the species being lumped together. Specifically, if the composite species $i$ is made up of individual species labeled by a second index $m$, so that subscript $i m$ refers to individual species $m$ within composite species $i$, then the coupling coefficients for the composite species pair $i j$ are given by

$$
\alpha_{i j}=\sum_{m n} \alpha_{i m, j n} \quad ; \quad \beta_{i j}=\sum_{m n} \beta_{i m, j n} \quad .
$$

and the charge per unit mass of composite species $i$ is given by

$$
q_{i}=\sum_{m} y_{i m} q_{i m}
$$

where $y_{i m}=\rho_{i m} / \rho_{i}$ is the mass fraction of individual species $m$ within composite species $i$.

\section{ACKNOWLEDGMENT}

I am grateful to Larry Cloutman for thoughtful comments on the manuscript and many helpful discussions.

\section{REFERENCES}

1. J. D. Ramshaw, J. Non-Equilib. Thermodyn. 18, 121 (1993).

2. J. D. Ramshaw and C. H. Chang, Phys. Rev. E 53, 6382 (1996).

3. J. D. Ramshaw and C. H. Chang, Plasma Chem. Plasma Process. 13, 489 (1993).

4. J. D. Ramshaw, J. Non-Equilib. Thermodyn. 21, 233 (1996).

5. J. O. Hirschfelder, C. F. Curtiss, and R. B. Bird, Molecular Theory of Gases and Liquids (Wiley, New York, 1954). 
6. S. Chapman and T. G. Cowling, The Mathematical Theory of Non-Uniform Gases, 3rd ed. (Cambridge U. P., Cambridge, 1970).

7. L. D. Cloutman, "A Selected Library of Transport Coefficients for Combustion and Plasma Physics Applications," Lawrence Livermore National Laboratory report (in preparation).

8. J. M. Burgers, Flow Equations for Composite Gases (Academic, New York, 1969).

9. S. I. Braginskii, "Transport Processes in a Plasma," in Reviews of Plasma Physics, Vol. 1, p. 205 (Consultants Bureau, New York, 1965).

10. M. Mitchner and C. H. Kruger, Partially Ionized Gases (Wiley, New York, 1973).

11. L. Spitzer, Jr., Physics of Fully Ionized Gases, 2nd rev. ed. (Interscience, New York, 1962).

12. J. D. Ramshaw, "Fluid Dynamics and Energetics in Ideal Gas Mixtures," Am. J. Phys. (submitted).

13. J. D. Ramshaw, J. Non-Equilib. Thermodyn. 15, 295 (1990).

14. J. D. Ramshaw, J. Non-Equilib. Thermodyn. 23, 135 (1998). 